\title{
Facies recognition using wavelet based fractal analysis and waveform classifier at the Oritupano-A Field, Venezuela
}

\author{
M. López and M. Aldana \\ Earth Science Department, Simón Bolívar University, Caracas, Venezuela \\ Received: 12 December 2006 - Revised: 15 May 2007 - Accepted: 22 June 2007 - Published: 3 July 2007
}

\begin{abstract}
We have used a Wavelet Based Fractal Analysis (WBFA) and a Waveform Classifier (WC) to recognize lithofacies at the Oritupano A field (Oritupano-Leona Block, Venezuela). The WBFA was applied first to Sonic, Density, Gamma Ray and Porosity well logs in the area. The logs that give the best response to the WBFA are the Gamma Ray and NPHI (porosity) logs. In the case of the logs, the lithological content could be associated to the fractal parameters: slope, intercept and fractal dimension. The map obtained using the fractal dimension shows tendencies that generally agree with the depositional patterns previously observed in conventional geological maps. According to the results obtained in this study, zones with fractal dimension values lower than 0.9 correspond to sandstone channels. Values between 0.9 and 1.2 coincide with the interdistributary deltaic shelf and values greater than 1.2 might be associated with zones of greater shale content. The WBFA and WC results obtained for the seismic data show no relation with the lithofacies. The lost of low and high frequencies in these seismic data, as well as phase problems, could be the reasons for this behavior.
\end{abstract}

\section{Introduction}

To obtain a reliable reservoir characterization, it is important to have a proper identification and mapping of rock facies. This means that reservoir characterization requires the lithological and petrophysical knowledge of the study area. In this sense, the identification of rock types is a main objective in oil exploration, in order to find reservoir and seal rocks (e.g. sandstones) (Álvarez et al., 2003). Facies identification and mapping are usually based on the inspection of core data and well logs (John et al., 2005). Statistical approaches have also been proposed for that purpose. John et al. (2005)

Correspondence to: $\mathrm{M}$. Aldana

(maldana@usb.ve) have used a simple form of the Bayes Theorem to compute the probability of occurrence of facies at different locations in a seismic cube, using seismic amplitude data. They use characters or patterns derived from seismic signals to identify facies. In this work we use the signature of the well logs and the seismic signals to recognize facies.

The spectral analysis of a time series is one of the techniques most widely used to obtain the series signature. This analysis decomposes the time series into a number of components, each one associated with a particular frequency. It is also possible to decompose the time series into a number of components that are associated with a particular scale at a particular time. This is known as the wavelet transform or wavelet analysis of a time series (Kumar and FoufoulaGeorgiou, 1994; Rioul and Vetterli, 1991). A signal can also be characterized by its fractal dimensions (Russell et al., 1980). As in the wavelet theory, with the fractal analysis the behavior of a signal can be characterized using scaling tools in the measurement. These two approaches have been previously combined to analyze time series. Argoul et al. (1989) have used the wavelet transform for a fractal description of images. Akay (1995) has applied a Wavelet Based Fractal Analysis (WBFA) to study biomedical signals. Using this combined analysis, important features of the signal have been extracted to understand or model physiological systems. Moreover, the scale property of the wavelet transform has provided a framework for studying the scale properties of seismic and reservoir data. In this sense, a 3D wavelet transformation of geostatistical reservoir data has been used to characterize the reflectivity scale spectrum and the relation between reflectivity at different scales in a reservoir (Mosher et al., 1998). Jiménez et al. (1999) have applied WBFA to study two wells, trying to identify lithology. They have found that plots of the logarithm of the variance of the wavelet coefficients versus scale discriminate a well, mainly sandy, from the other with a major content of shale.

In the present work we have used a Wavelet Based Fractal

Published by Copernicus Publications on behalf of the European Geosciences Union and the American Geophysical Union. 
Analysis (WBFA) and a Waveform Classifier (WC) to recognize lithofacies at the Oritupano A field, Venezuela, in both well logs and seismic traces. The study area presents paleosedimentary characteristics that indicate a variation from marine-coastal environments to estuarine or deltaic ones. A variation in the sand/shale content is also expected. We try to identify the facies at the field by using the parameters derived from the WBFA and a fractal dimension obtained from them. Maps of these parameters could show the general behavior of lithofacies in the area. For seismic data, WBFA and WC results will be compared.

\section{Wavelet Based Fractal Analysis (WBFA)}

The wavelet method transforms a given time series to a scaletime domain. This transform is performed by correlating the time series $f$ with a shifted and translated function $\varphi$, according to:

$C(a, e)=\frac{1}{\sqrt{a}} \int_{-\infty}^{\infty} f(t) \overline{\left(\frac{t-e}{a}\right)} d t ; a, e \in \Re, a>0$

The function $\varphi$ is called a wavelet. The wavelet is a function with limited energy and with zero mean (admissibility condition). The wavelet coefficients $C(a, e)$ give information about the scale $a$ as well as about the time $e$ of appearance of a characteristic structure (Kumar and Foufoula-Georgiou, 1994).

The time series $f(t)$ can be recovered from its wavelet transform $C(a, e)$ by:

$$
f(t)=\frac{1}{C_{\varphi}} \int_{-\infty}^{\infty} \int_{0}^{\infty} C(a, e) \overline{\varphi\left(\frac{t-e}{a}\right)} \frac{\text { dade }}{a^{2}}
$$

$\mathrm{C}_{\varphi}$ is called the admissibility constant and is given by

$$
C_{\varphi}=2 \pi \int_{0}^{\infty} \frac{|\hat{\varphi}(\omega)|^{2}}{|\omega|} d \omega
$$

where $\hat{\varphi}$ is the Fourier transform of $\varphi$. The admissibility constant has to satisfy the admissibility condition:

$$
0<C_{\varphi}<+\infty
$$

This transform has found a lot of applications not only in signal processing, but also in the geophysical sciences (Kumar and Foufoula-Georgiou, 1994).

Akay (1995) has proposed the use of a Wavelet Based Fractal Analysis (WBFA) in order to obtain the dimension of the heart-sound waveforms. For this analysis, the variance of the wavelet coefficients is obtained, at each level of decomposition, and the log-variance plotted against the scale. Linearity regions in these plots correspond to a power-law process over a particular frequency region. The slope of the line can be related with the exponent of the power-law process (Percival and Guttorp, 1994).

Based on the potential definition of the fractal dimension (Mandelbrot, 1977), Berry (1979) has obtained, for a one dimensional profile, a relationship between the slope of a graphic of the variance of the power spectrum (given by the Fourier Transform) vs. frequency and the fractal dimension:

$m=5-2 D$

where $m$ is the slope of the linear region and $D$ is the fractal dimension. An alternative approach to this relationship has been given by Mengesha (1999). Considering the equivalence between Fourier and Wavelet transforms, we have used equation (5) to obtain the fractal dimension associated to the plots of $\log$-variance of the wavelet coefficients $(\log (\sigma))$ versus scale. It is important to notice that this dimension just characterizes the time series (well log or seismic trace) that could be associated with different sedimentary environments.

\section{Waveform Classifier (WC)}

The variations in the character of a seismic reflector or a set of reflectors might be associated to changes in the strata geometry or lithologic stacking patterns. Hence, in seismic stratigraphy, the recognition of those systematic variations is an important task (Hall and Trouillot, 2004). This kind on analysis could help to elucidate subsurface stratigraphy qualitatively and also quantitatively when is coupled with seismic modeling. The recognition of these variations, via the comparison of the wavelets shape, is called waveform classification.

There are two main approaches to waveform classification: supervised and unsupervised. In a supervised mode, a wavelet of a zone of interest is used to guide the grouping of similar waveforms in the whole area and mapping them. The searching is performed at a time window. The main assumption is that the source wavelet is similar for all traces, and that the waveform of similar traces, adjacent or not, is in part the result of the same stratigraphy (Ross and Peterman, 2000). Hence, the spatial variation of the waveform could be an indicator of different geological features. The results of the classification process can be displayed in a map of wavelet classes in which each class is represented by an integer. In the unsupervised classification, a reference wavelet is automatically calculated from the reference data before assigning classes. This assignation is achieved after an iterative statistical analysis of a subset of the data; the results obtained are then applied to the entire dataset. Again, the results of the classification process can be displayed in a map of wavelet classes. This approach is useful if no a priori information (e.g. correlation with wells or even well information) is available (Hall and Trouillot, 2004).

We have performed a supervised waveform classification of the 3-D seismic data of the area, using the Waveform 
Classifier (WC) provided by Landmark Graphics Corporation (Haber and Wilk, 2006). The analyses were carried out in a window around the interpreted horizon. The numerical differences in the wavelets were computed using the Manhattan distance (see for example Tielen et al., 1997). The Manhattan distance function computes the distance that would be traveled to get from one data point to the other if a grid-like path is followed. The Manhattan distance between two items is the sum of the differences of their corresponding components. The difference between two wavelets, $\phi$ and $\psi$, with $N$ samples, is measured using this distance as:

$\Delta=\sum_{i=1}^{N}\left|\varphi_{i}-\psi_{i}\right|$

Two identical wavelets will give $\Delta=0$, whereas different wavelets will give positive $\Delta$ values. These values are used to obtain the WC maps.

\section{Geographical and geological setting of the Oritupano- A field}

The Oritupano-Leona Block is located at the Greater Oficina Trend (see Fig. 1). More than 12 isolated oil fields constitute the block. Structurally, the fields are located in the southern flank of the Eastern Venezuela Basin, in the foredeep platform zone (Parnaud et al., 1995). The Oritupano A field is affected by normal faulting mainly trending $\mathrm{N} 60^{\circ} \mathrm{E}$. The sedimentary sequence is conformed by coarse to medium sandstones interbedded with shales and cherts. The productive section is represented by the Oficina Formation (Early Miocene) (see Fig. 2), although the uppermost reservoirs of the underlying Merecure Formation (Oligocene) have produced an acceptable oil volume. The cumulative oil production of the block is over $340 \mathrm{MMbbls}$ since its discovery in the 1940's. Reservoirs are composed of sandstones of estuarine to shallow-marine environments (Porras et al., 2002). We have studied the lower unit of the Oficina Formation, known as the LU hydraulic unit. This unit was divided in eight layers: LM2, L3, M2, M4, O1, P2-3, R1 and U1.

A paleosedimentary study was previously performed in the area (Grosso, 2002) and extended in this work. Facies maps that reflect the sedimentary tendencies, lithology and reservoir quality for each layer of the LU unit were obtained. Those wells with core analysis were classified as A and B based on the paleoenvironments identified, the quality of the reservoir and the sand/shale content (calculated from the Gamma Ray logs). Wells A are characterized by the presence of clean thick sands, good quality reservoirs and are mainly associated with one paleoenvironment (see Fig. 3a, well ORM-117). In wells B, more than one sedimentary environment could be interpreted (e.g. marine-coastal, estuarine and deltaic); the reservoir quality ranges from regular to bad and the predominant lithology is shale (see Fig. 3b, well

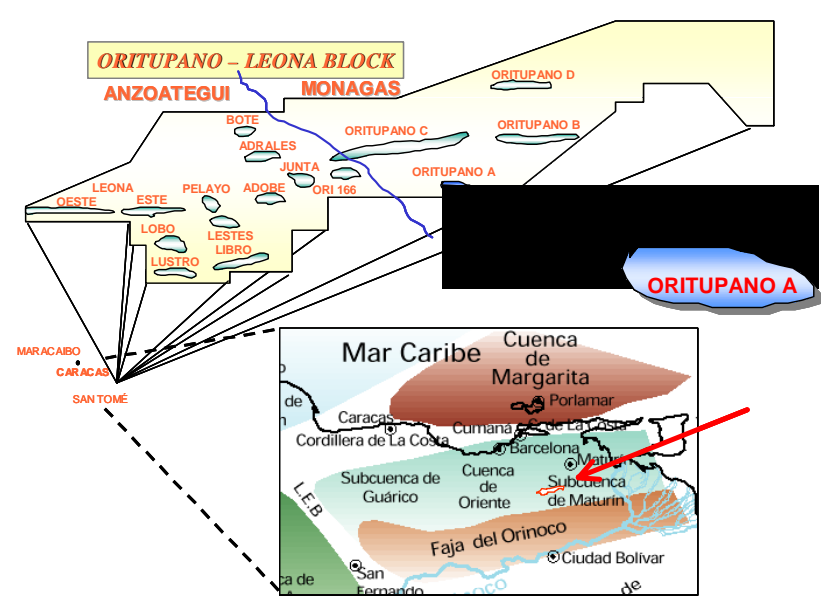

Fig. 1. Geographical setting of the study area.

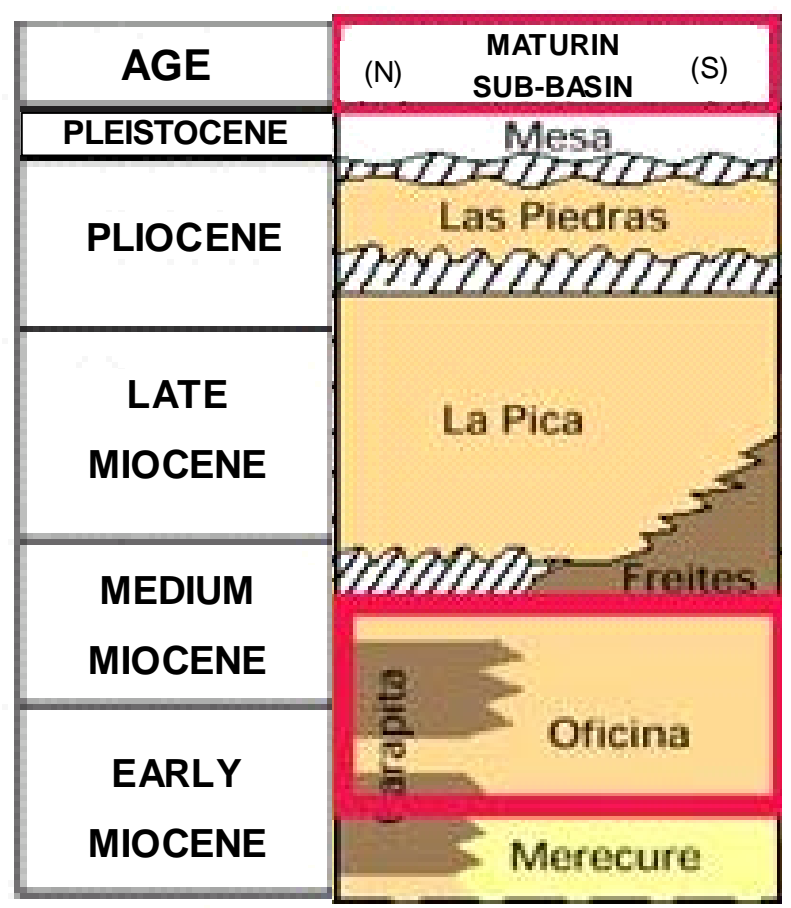

Fig. 2. Stratigraphic column of the study area.

ORM-94). Table 1 shows the result of the classification of 18 wells.

A map of net sand was also obtained for the study area (see Fig. 4). Zones of great thickness are observed. These zones can be associated to channels. Also this map shows sedimentary and distribution patterns that can be associated to an interdistributary zone of a deltaic plain. 

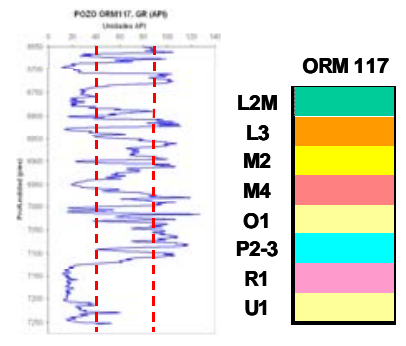

a)
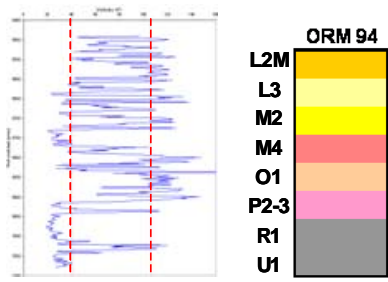

b)

\begin{tabular}{|c|c|c|c|c|}
\hline & $\begin{array}{c}\text { Reservoir } \\
\text { Quality }\end{array}$ & Architecture & Lithology & Paleoenvironment \\
\hline & Very Good & Shoreline-bar & Coarse Sand & Marine-Coastal \\
\hline \hline & Good & Tidal bar & Sand-Coal & Marine-Coastal \\
\hline \hline & Good-Regular & Tidal bar & Medium Sand & Marine-Coastal \\
\hline & Good & Channel & Coarse Sand & Deltaic \\
\hline \hline & Very Good & Channel & Coarse Sand & Deltaic \\
\hline \hline & Regular & Tidal bar & Medium Sand & Marine-Coastal \\
\hline & Regular & Channel & Medium Sand & Estuarine \\
\hline & Regular-Bad & Tidal bar & Sand-Coal & Marine-Coastal \\
\hline & Regular & Channel & Medium Sand & Estuarine \\
\hline & Bad & Bar & Siltstone & Marine-Coastal \\
\hline \hline & Bad & Channel & Siltstone & Marine-Coastal \\
\hline \hline & Bad & Swamp & Coal-Lignite-Silt-Shale & Swamp \\
\hline & No reservoir & Off shore & Shale & Deep Marine \\
\hline
\end{tabular}

Fig. 3. (a) GR $\log$ and results of the paleosedimentary study of well ORM-117. This well was classified as A; (b) GR log and results of the paleosedimentary study of well ORM-94. This well was classified as B; and (c) Reference table of the paleosedimentary study indicating, by color, the architecture, lithology and paleoenvironment.

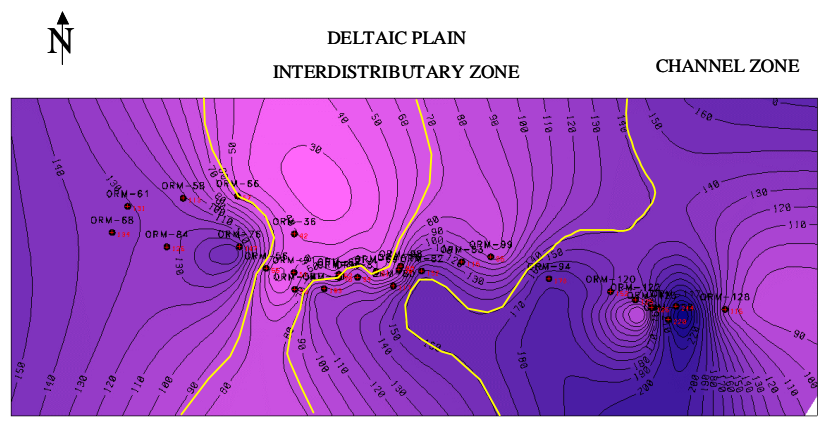

Fig. 4. Net sand map of the study area, showing the interdistributary and channel zones.
Table 1. Well classification based on the paleosedimentary study and the sand/shale content.

\begin{tabular}{ll}
\hline Well & Well Type \\
\hline ORM-76 & A \\
ORM-82 & A \\
ORM-83 & A \\
ORM-117 & A \\
ORM-78 & A \\
ORM-142 & A \\
ORM-158 & A \\
ORM-123 & A \\
ORM-80 & A \\
ORM-36 & B \\
ORM-75 & B \\
ORM-61 & B \\
ORM-84 & B \\
ORM-66 & B \\
ORM-94 & B \\
ORM-105 & B \\
ORM-81 & B \\
ORM-68 & B \\
\hline
\end{tabular}

\section{Well analysis}

A group of 12 wells among all the wells in the area was selected to test a set of wavelets for the WBFA. This set includes wavelets from Biorthogonal, Symlet, Coiflet, Morlet, Meyer, Mexican Hat, Haar and Daubechies families (Foufoula-Georgiou and Kumar, 1994). The WBFA was performed on the available well logs of the test group (Gamma Ray (GR), Sonic, Density and Porosity (NPHI) logs). The decomposition process was applied in the depth range that comprises the hydraulic unit of interest (LU). This process was iterated, with successive approximations decomposed in turn, so that one signal was broken down into many lowerresolution components (wavelet decomposition tree). A clear linear tendency was observed in all the cases in the graphs of the logarithm of the variance of the wavelet coefficients $(\log (\sigma))$ versus scale. The best decomposition was achieved with six levels for most of the logs. To perform the WBFA for the whole area, we have selected the wavelet that provided the better linear adjustment in these graphics, based on the linear regression coefficient, $\mathrm{R}^{2}$. For each well and each type of log, a plot of the $\mathrm{R}^{2}$ values associated with the tested wavelets was obtained. Figure 5a shows one of these graphs for a NPHI log. After analyzing all these plots, the best adjustment, based on the $\mathrm{R}^{2}$ coefficient, was obtained with a wavelet from the Biorthogonal family (see Fig. 5a). Finally, the WBFA with the selected wavelet was performed on 32 wells available in the area at the interval of interest.

The variance of the wavelet coefficients, $\sigma$, was calculated and plotted versus the scale in a semi-log plot. Figure $5 \mathrm{~b}$ 


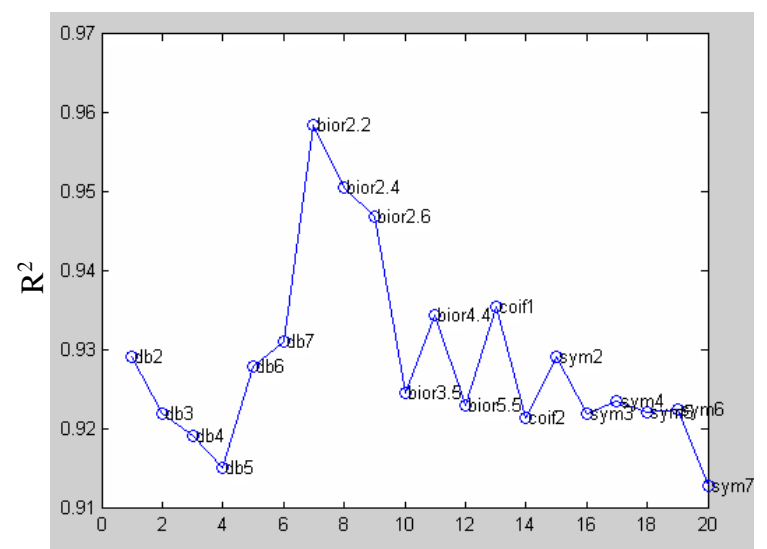

a)

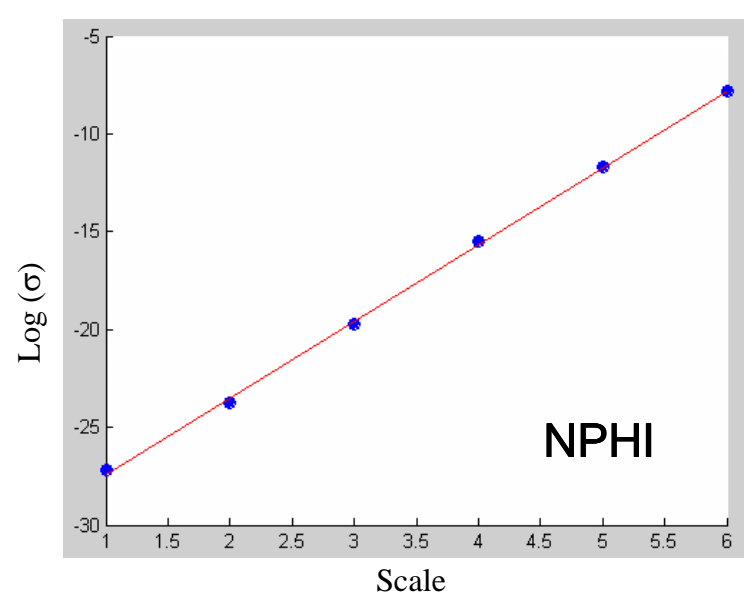

b)

Fig. 5. (a) Linear regression coefficients, $R^{2}$, obtained for the linear region of the logarithm of the variance $(\sigma)$ of the wavelet coefficients versus scale plots, after decomposing a NPHI log with wavelets from different families: Biorthogonal (bior), Symlet (sym), Coiflet (coif) and Daubechies (db). The best result is obtained for a wavelet of the Biorthogonal family. (b) $\log (\sigma)$ versus scale for a NPHI log, after decomposing it with a Biorthogonal wavelet.

shows the results obtained for the NPHI log of one of the studied wells. A fine linear fit is obtained; similar results were observed for the rest of the wells and the logs. From these plots, the slope $m$ and the intercept $b$ were calculated.

As a first step, we studied the dependence of the $m$ and $b$ values with the well log type. Only 12 of the total number of wells in the area have a complete set of logs that includes GR, Sonic, Density and NPHI. Figure 6 shows the results obtained for this set of wells. As can be observed, the $m$ value varies within a range for all the logs analyzed. Nevertheless, the $b$ value depends on the well log type and set them apart, namely GR and Sonic logs are associated with lower absolute $b$ values compared with Density and NPHI logs. This was expected as diverse logs respond in a different way, that is they have a different waveform, even in the same lithological

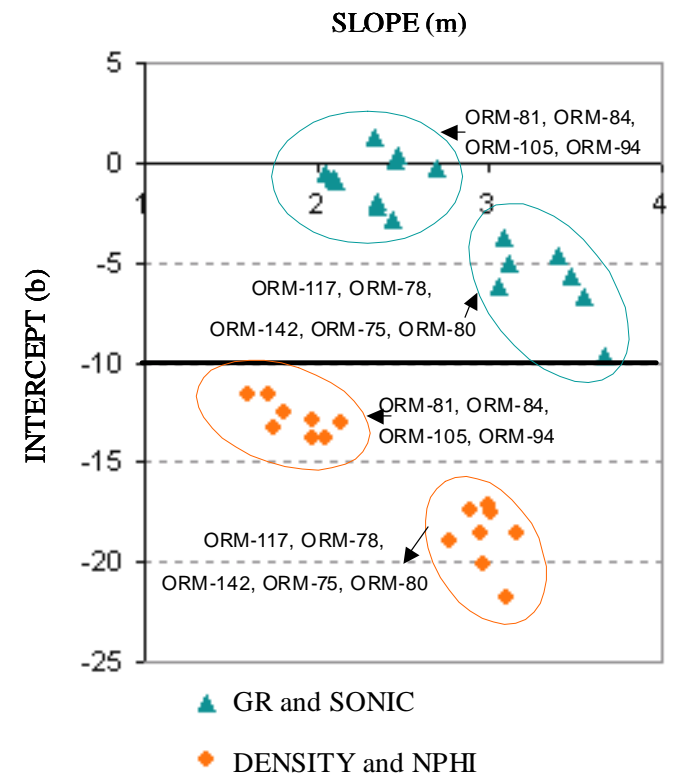

Fig. 6. $m$ and $b$ values for different logs. Only those wells with a complete set of logs (GR, Sonic, NPHI and Density) were included in this graph.

zone. This kind of graphic also sets apart wells with more sand content from those with more shale content. Greater absolute values of $m$ and $b$ correspond to wells associated with just one depositional environment, with good reservoir quality, and with considerable clean sands (e.g. ORM-117, ORM-78, ORM-142 and ORM-80). Lower absolute values of $m$ and $b$ are associated with the presence of shale layers, with interbedded thinner sand layers and medium reservoir quality (e.g. ORM-81, ORM-84, ORM-105 and ORM-94). This separation is clearer for Density and NPHI logs.

The $m$ and $b$ values obtained for the GR and NPHI logs of all the wells studied in the area are presented in Table 2 and plotted in Fig. 7. Instead of just a two groups separation, a linear behavior is observed for both kinds of logs in this graphic. The tendency is in agreement with the lithological content of each well, as can be observed in Fig. 8, where some representative GR logs have been inserted for some data points of Fig. 7. Wells with more content of sand layers, that correspond to channel zones, have higher values of $m$ (e.g. ORM-76). Wells with more content of shales have lower values of $m$ (e.g. ORM-91). The middle section of the line includes $m$ and $b$ values that characterize wells with alternations of sands and shales, and some of them are located in the interdistributary zone of the deltaic plain (e.g. ORM-36). Then, in this case, not only two end members (i.e. with mainly sand or mainly shale content) are observed, but a gradual change of lithological content has been characterized using $m$ and $b$ values derived from the WBFA analysis.

Using the slope $m$ of the plots of $\log (\sigma)$ vs. scale (see 


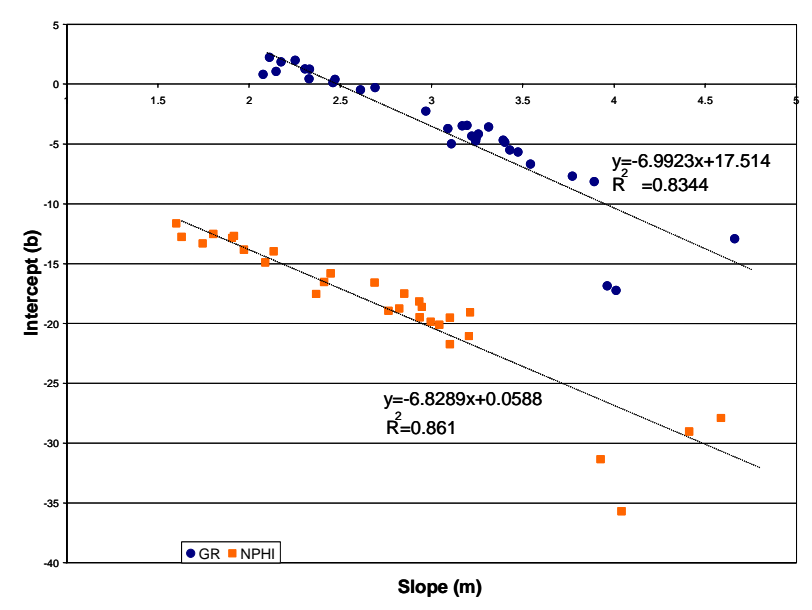

Fig. 7. $m$ and $b$ values obtained after the WBFA of the GR and NPHI logs of all the studied wells in the area. The equations and $\mathrm{R}^{2}$ values of the lines that follow the observed tendencies of the data are also included.

Fig. 5b), a fractal dimension D can be calculated via Eq. (5). The D values obtained from the GR logs of all the wells are presented in Fig. 9 and Table 2. The values were plotted in an increasing way. At least, four different groups, indicated by different slopes of the tendency lines that connect the data, can be observed in Fig. 9a. In Fig. 9b. representative GR logs have been inserted. Also a concise description of the main environment characteristics, from previous works (Grosso, 2002), is provided in this figure. The most important result is that the $\mathrm{D}$ values sort out the groups of wells according to the lithology. In other words, there is a relationship between fractal dimension and lithology, as is explained below.

As can be observed from Table 2, values of $\mathrm{D}$ lower than 0.9 were obtained for wells A. These wells are associated to high energy sedimentary environments, channels and thick sands of medium to coarse grains (see Fig. 9b). Values of D greater than 1.2 were obtained for those wells associated to sections with higher shale content, low energy environments or marsh zones (see Fig. 9b). The wells located at the interdistributary zone identified via the net sand map (see Fig. 4) have values of $\mathrm{D}$ between 0.9 and 1.2 (wells ORM-36, ORM80, ORM-75 and ORM-66).

A fractal dimension D map was obtained for the study area (Fig. 10). This map can be compared with the net sand map of the area (Fig. 4). In the D map, different zones can be interpreted, and some of the patterns observed are in agreement with those features previously interpreted in the net sand map. Lower fractal dimensions $(<0.9)$ are observed to the East and correspond to the channel zone identified in the net sand map (great sand thickness layers; e.g. ORM-117). Values between 0.9 and 1.2 correspond to the interdistributary zone of deltaic plain also identified in the net sand map. Wells in this zone show a transitional behavior with thinner
Table 2. $m, b$ and D values obtained after the WBFA of the GR logs. The classification of the well as A or B (see Table 1) is also indicated ( $\mathrm{NC}=$ Not classified).

\begin{tabular}{lllll}
\hline Well & $\mathrm{m}$ & $\mathrm{b}$ & $\mathrm{D}$ & Well Type \\
\hline ORM-128 & 4.6618 & -12.9252 & 0.1691 & $\mathrm{NC}$ \\
ORM-76 & 4.0113 & -17.229 & 0.49435 & $\mathrm{~A}$ \\
ORM-82 & 3.9625 & -16.8549 & 0.51875 & $\mathrm{~A}$ \\
ORM-54 & 3.8919 & -8.1412 & 0.55405 & $\mathrm{NC}$ \\
ORM-83 & 3.7716 & 7.6857 & 0.6142 & $\mathrm{~A}$ \\
ORM-117 & 3.543 & -6.6758 & 0.7285 & $\mathrm{~A}$ \\
ORM-78 & 3.4732 & -5.669 & 0.7634 & $\mathrm{~A}$ \\
ORM-56 & 3.4293 & -5.5037 & 0.78535 & $\mathrm{NC}$ \\
ORM-95 & 3.4015 & -4.8482 & 0.79925 & $\mathrm{NC}$ \\
ORM-142 & 3.3926 & -4.6788 & 0.8037 & $\mathrm{~A}$ \\
ORM-73 & 3.3128 & -3.5737 & 0.8436 & $\mathrm{NC}$ \\
ORM-122 & 3.2576 & -4.1634 & 0.8712 & $\mathrm{NC}$ \\
ORM-158 & 3.2433 & -4.5365 & 0.87835 & $\mathrm{~A}$ \\
ORM-123 & 3.2422 & -4.7537 & 0.8789 & $\mathrm{~A}$ \\
ORM-120 & 3.2199 & -4.3394 & 0.89005 & $\mathrm{NC}$ \\
ORM-67 & 3.194 & -34.404 & 0.903 & $\mathrm{NC}$ \\
ORM-36 & 3.1688 & -3.4921 & 0.9156 & $\mathrm{~B}$ \\
ORM-80 & 3.1092 & -4.9827 & 0.9454 & $\mathrm{~A}$ \\
ORM-75 & 3.0896 & -3.7088 & 0.9552 & $\mathrm{~B}$ \\
ORM-61 & 2.9686 & -2.2577 & 1.0157 & $\mathrm{~B}$ \\
ORM-84 & 2.6909 & -0.2928 & 1.15455 & $\mathrm{~B}$ \\
ORM-66 & 2.6109 & -0.4823 & 1.19455 & $\mathrm{~B}$ \\
ORM-94 & 2.4699 & 0.3868 & 1.26505 & $\mathrm{~B}$ \\
ORM-105 & 2.4599 & 0.1166 & 1.27005 & $\mathrm{~B}$ \\
ORM-81 & 2.3308 & 1.2519 & 1.3346 & $\mathrm{~B}$ \\
ORM-58 & 2.3293 & 0.4398 & 1.33535 & $\mathrm{NC}$ \\
ORM-99 & 2.3053 & 1.2761 & 1.34735 & $\mathrm{NC}$ \\
ORM-98 & 2.2533 & 1.997 & 1.37335 & $\mathrm{NC}$ \\
ORM-91 & 2.1749 & 1.8659 & 1.41255 & $\mathrm{NC}$ \\
ORM-68 & 2.1475 & 1.0702 & 1.42625 & $\mathrm{~B}$ \\
ORM-69 & 2.1111 & 2.2387 & 1.44445 & $\mathrm{NC}$ \\
ORM-31 & 2.076 & 0.8258 & 1.462 & $\mathrm{NC}$ \\
\hline
\end{tabular}

sand and shale layers interbedded, and with similar net sand and shale content (e.g. ORM-66). Higher D values correspond to wells with more shale content, located to the West and Central zones of the area (e.g. ORM-94).

Jiménez et al. (1999) have applied the WBFA in the study of two wells, trying to identify lithology. They have found that plots of the logarithm of the variance of the coefficients vs. scale discriminate a well, mainly sandy, from the other with a major content of shale. In their work, they do not calculate the fractal dimension associated with these plots. Nevertheless, if the fractal dimension calculated via Eq. (5) is obtained for the two wells studied by Jimenez et al. (1999), also greater $\mathrm{D}$ values correspond to the mainly shaly environment.

It is important to notice that Jiménez et al. (1999) have performed a study of just two wells, and they have only found a 


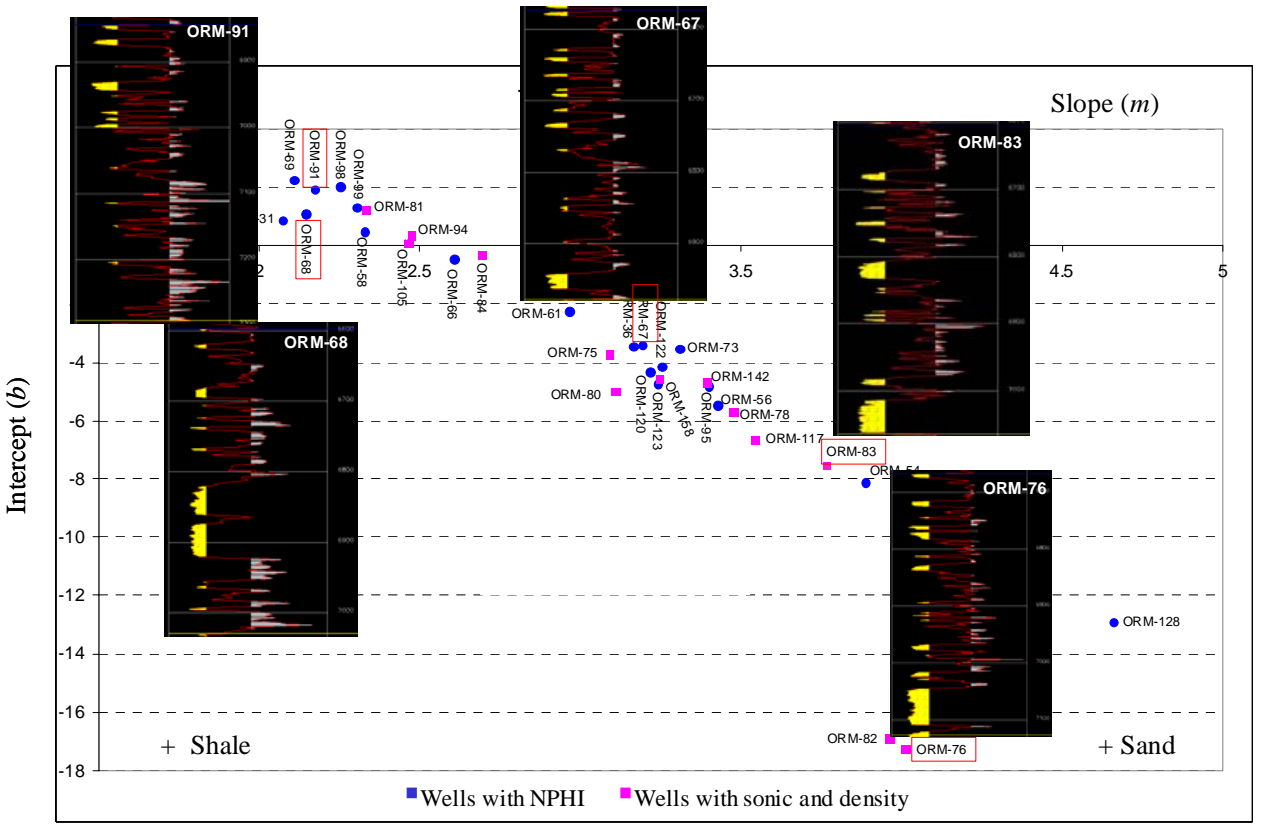

Fig. 8. $m$ and $b$ values obtained after the WBFA of the GR logs of all the studied wells in the area. Some representative GR logs are included.

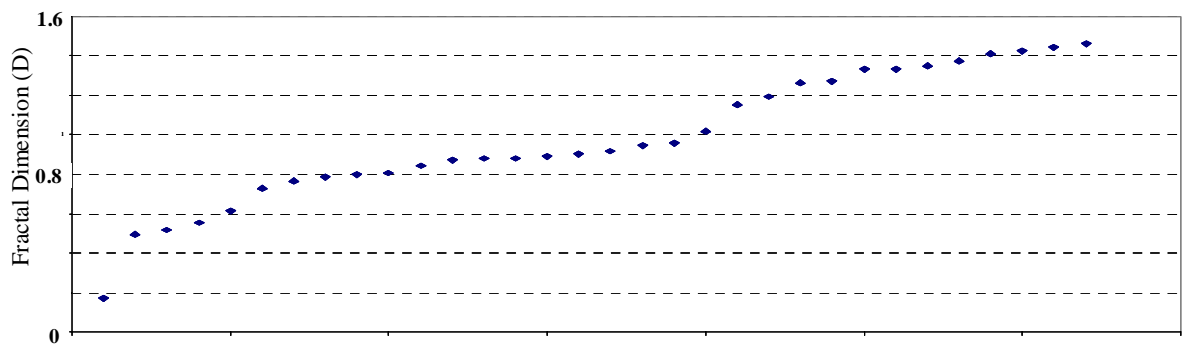

a)

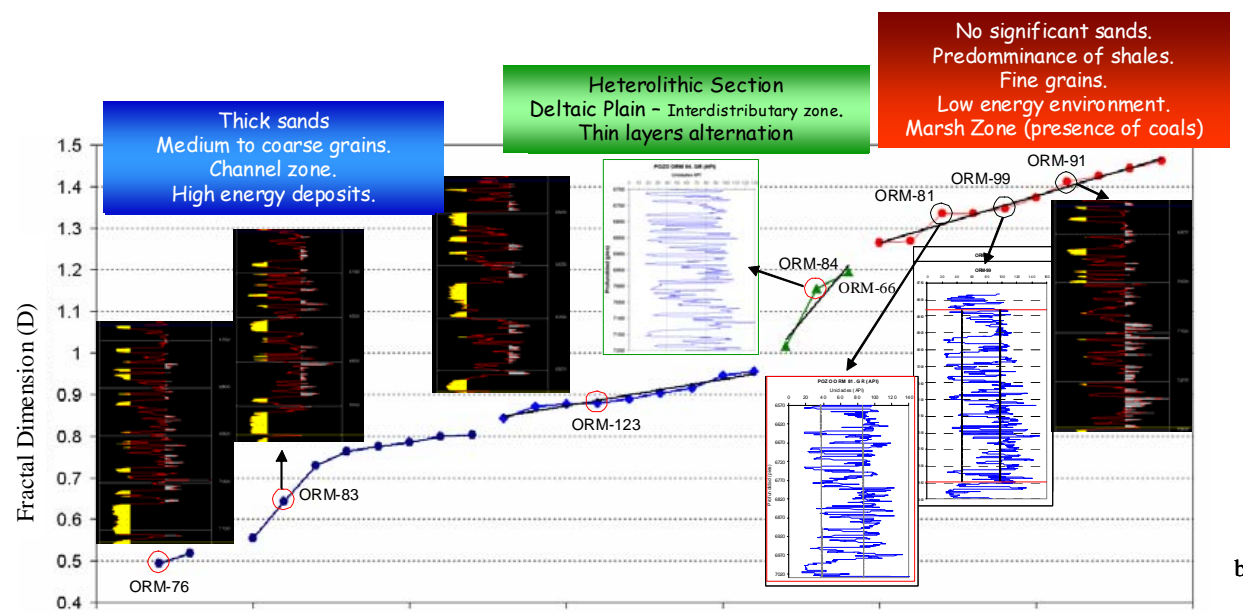

Fig. 9. (a) Fractal dimension distribution for the hydraulic unit LU; the D values were obtained after the WBFA analysis of the GR logs. (b) Some representative GR logs have been included. 
$\hat{\mathrm{N}}$

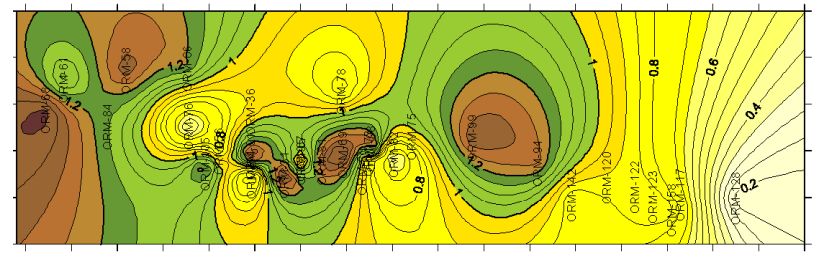

Fig. 10. Map of D values for the study area.

two end member classification, between a shaly and a sandy well. In the present work, after applying the method to a statistically significant number of wells, we have found that it is possible to identify, using the fractal dimension associated with the WBFA of the studied wells, not just two end members, but a whole range variation of shale and sand content. In fact, in this case we have obtained a D range that shows the transitional change between sandy and shaly environments. This variation corresponds to a gradual transition between different sedimentary environments. The maps obtained from the seismic data by Jiménez et al. (1999) in the area they have studied, just classified different zones as white or black (i.e. sandy or shaly). The fractal dimension maps that we have obtained in the present study allowed us to classify transitional environments and to observe, for example, stratigraphic features as channel or interdistributary zones in the study area. Hence, this kind of maps suggests that the fractal dimension can be used as a well log attribute or even a post-stack seismic attribute (Brown, 2004) for reservoir characterization.

Spectral techniques have been previously applied to analyze the fractal behavior of time series. Scale invariant power spectra for diverse well logs have been observed (e.g. Todoeschuck et al., 1990). Wavelet analysis has also been used to determine the frequency components of different time series (e.g. shoreline change signals, Tebbens et al., 2002). As in our case, when the relationship between variance and scale is well described by a power law, the studied signal is nonstationary and a self-affine fractal (Tebbens et al., 2002). The fractal dimension we have obtained in this work basically characterizes the analyzed logs. As it is generally known, greater fractal dimensions are associated with more complex sets (Turcotte, 1997). In our case the values of these fractal dimensions increase with the complexity of the studied logs. Less complex logs, associated with sandy environments, show more correlation between adjacent values (as the sand thickness increases) and smoother profiles. Consequently the fractal dimension values are lower. The behavior observed in well logs as those studied here (e.g. GR) is mainly associated with the lithological content of the area. Hence, a fractal based model for sedimentary basins (Hewett, 1998) or for sedimentation processes (Pelletier and Turcotte,

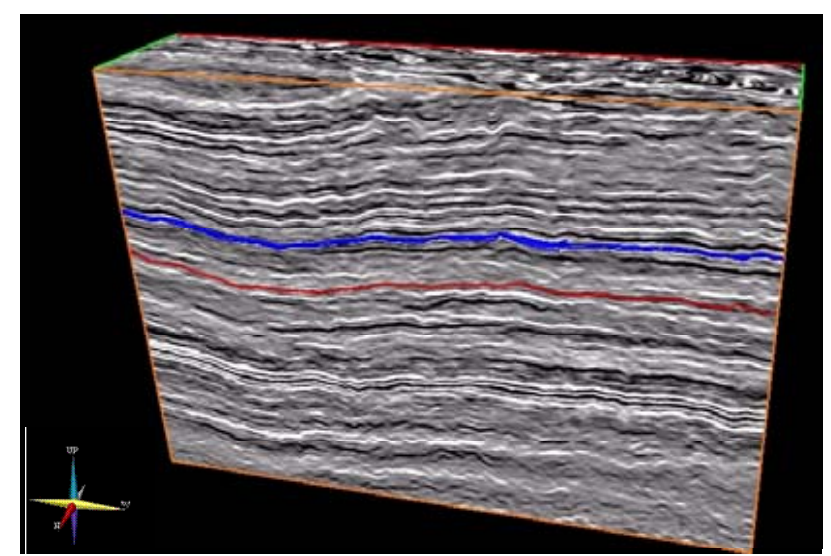

Fig. 11. Seismic cube of the study area. The unit of interest is located between 1600 and $1700 \mathrm{~ms}$, between the horizons Bur4 (blue) and Bur2 (red).

1996) could be expected. Hewett (1986), for example, has shown that variations in vertical porosity well logs from a submarine fan were scale invariant; namely, the power spectrum followed a power law dependence on the wavenumber. Based on this result, he developed a fractal based interpolation scheme in order to determine porosity variations from well logs in sedimentary basins and constructed realistic sedimentary structures (Pelletier and Turcotte, 1996). Schlager (2004) has indicated that the sediment architecture is largely scale invariant over a wide range of scales in time and space. He has also pointed out that first-order trends of sea-level movements and sedimentation rates are fractal on all geologically relevant time scales. These facts, that suggest a fractal behavior in the sedimentation process, could explain the results obtained in our work; namely, a relationship between a sedimentary environment, reflected by the well logs behavior, and a particular fractal dimension, according to the explanation given above. Nevertheless the statement of a particular sedimentation model is beyond the scope of this work.

\section{3-D seismic data analysis}

A 3-D post-stack migrated cube $\left(30 \mathrm{~km}^{2}\right)$ was used in this study (see Fig. 11). The unit of interest is located between 1600 and $1700 \mathrm{~ms}$ (between the horizons Bur4 (blue) and Bur2 (red) in Fig. 11). A combination of dynamite and vibroseis sources was used for this survey. WBFA and WC analysis were performed on a window around the studied horizon.

\subsection{WBFA}

To apply the WBFA on the seismic data, a seismic trace was extracted near each of the 32 wells of the study area. The analysis was applied on each extracted trace, following the same procedure used for the well logs. Again, for most of 


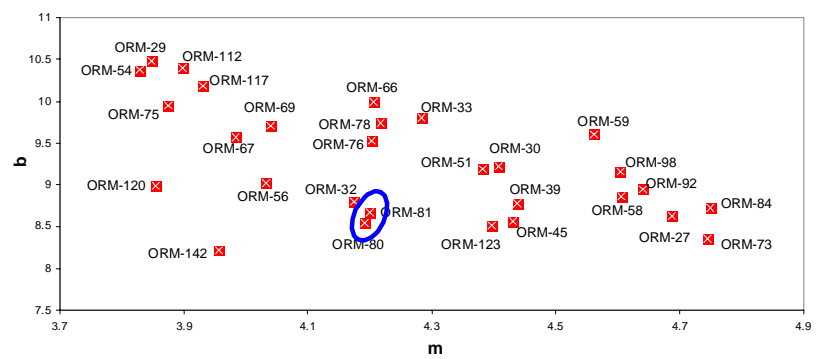

Fig. 12. $m$ and $b$ values obtained from the WBFA analysis of the closest seismic trace to each of the studied wells in the area. The blue oval enclosed wells ORM-81 and ORM-80 which have different lithologies.

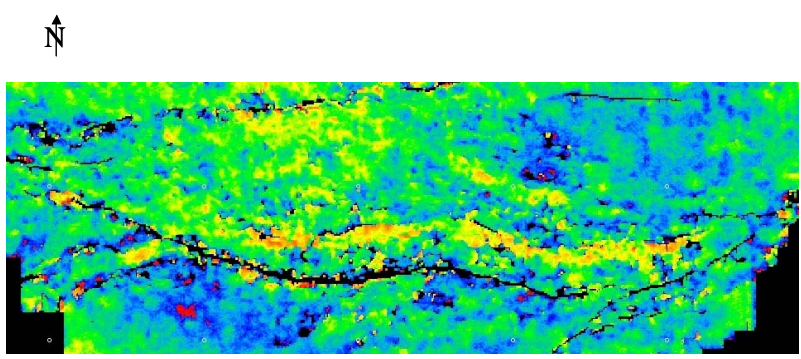

Fig. 13. Phase map of the study area. The colors correspond to the phase range: yellow: $0^{\circ}$ to $15^{\circ}$; green: $15^{\circ}$ to $45^{\circ}$; blue: $45^{\circ}$ to $80^{\circ}$, and red: $80^{\circ}$ to $115^{\circ}$.

the traces, the best fit was achieved with a wavelet from the Biorthogonal family and with six levels. The obtained $m$ and $b$ values were represented in a plot similar to that of Fig. 7 (see Fig. 12). In this case, there is no tendency or clustering according to the facies or even to the lithological content. No grouping between traces that correspond to similar sedimentary environments was obtained, although the logs of the wells near these traces respond to the lithological variation. In fact, the seismic traces extracted close to the wells ORM81 and ORM-80 (enclosed in the oval of Fig. 12) have nearly the same values of $b$ and $m$; nevertheless, the GR logs of these two wells indicate dissimilar lithologies for these locations, i.e. sandy environment at ORM-80 and shaly at well ORM-81 (see Table 2).

It is clear that the WBFA results obtained for the seismic cube are completely different to those obtained for the well logs. The fractal parameters obtained after the WBFA of the well logs seem to respond to the lithological variation in the study area; this is not the case for the seismic data, as was discussed above.

A possible explanation for these results could be a phase problem that was observed in the 3-D seismic data and the lack of low $(0-16 \mathrm{~Hz})$ and high $(80-200 \mathrm{~Hz})$ frequencies in these data (Aristimuño and Aldana, 2006). The phase problem is illustrated in the map of Fig. 13. Phases ranging from
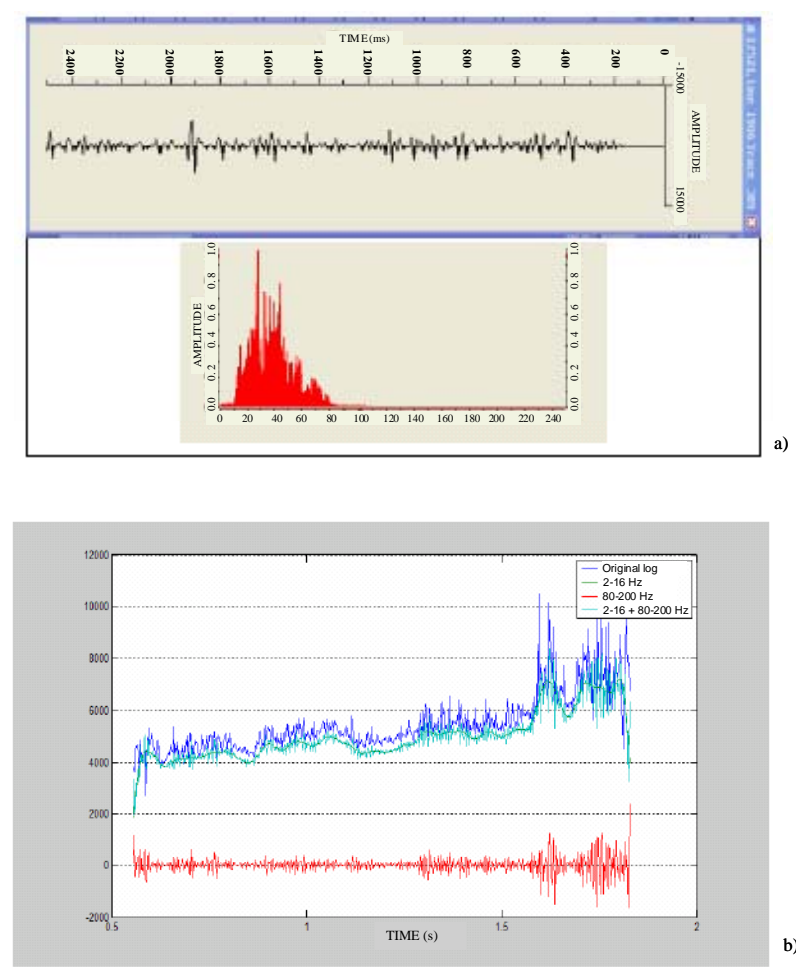

Fig. 14. (a) Representative frequency spectrum obtained for the seismic data analyzed in this work (after Aristimuño and Aldana, 2006) (b) Original well log (dark blue) and filtered logs with different frequency ranges content: $2-16 \mathrm{~Hz}$ (green), $80-200 \mathrm{~Hz}$ (red) and $2-16 \mathrm{~Hz}+80-200 \mathrm{~Hz}$ (light blue) (after Aristimuño and Aldana, 2006).

0 to 115 degrees can be observed. Just processing the data to get the same phase is not an easy task as the phase variation was introduced in the acquisition process by the combination of vibroseis (zero phase) and dynamite (minimum phase) sources. On the other hand, the frequency spectrum of seismic data is always narrower than that of well logs. Generally, frequencies above $100 \mathrm{~Hz}$ are lost in the acquisition process of seismic data. A representative frequency spectrum obtained for the seismic data analyzed in this work is presented in Fig. 14a. As can be observed, the frequencies range from 16 to $80 \mathrm{~Hz}$. Analyses performed by Aristimuño and Aldana (2006) indicate that the lithological response observed in the well logs of the study area could be associated mainly to the low $(0-16 \mathrm{~Hz})$ and high $(80-200 \mathrm{~Hz})$ frequency ranges, that are not observed in the seismic data. This behavior is illustrated in Fig. 14b (after Aristimuño and Aldana, 2006) where different band-pass filters are applied to a log of one of the studied wells and the results are compared with the original one. As can be observed, the log with the frequency content that ranges from 2 to $16 \mathrm{~Hz}$ nearly resembles the general behavior or wave form of the original $\log$. The addition of the filtered logs with frequencies from 


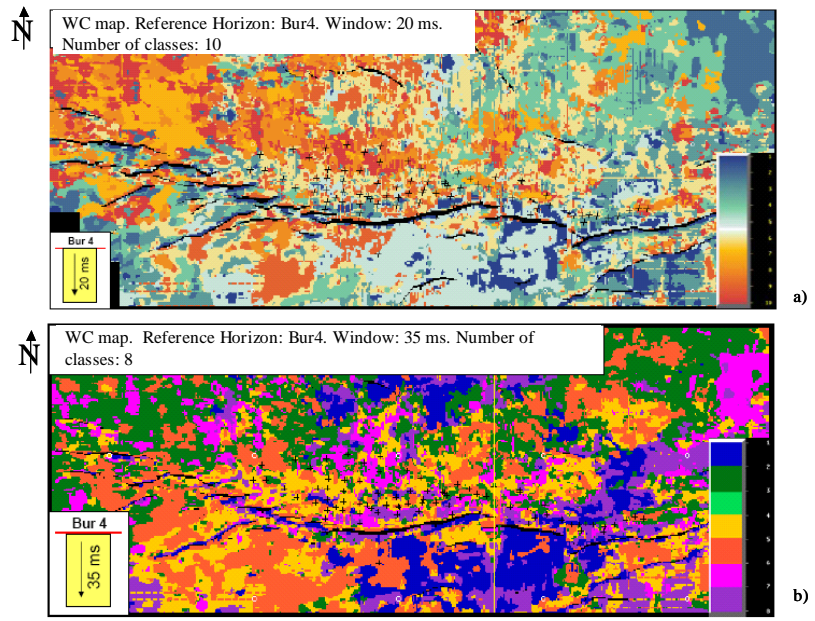

Fig. 15. Waveform Classifier maps, using as reference horizon Bur4: (a) Window: $20 \mathrm{~ms}$, Number of Classes: 10; (b) Window: $35 \mathrm{~ms}$, Number of Classes: 8 .

0 to $16 \mathrm{~Hz}$ and 80 to $200 \mathrm{~Hz}$ completely reproduce the original log. These frequency ranges are absent in the seismic data. Hence, no appropriate lithological information could be derived from a WBFA analysis of these data, as the main associated waveforms are not present. It is important to point out that these results do not preclude the use of WBFA to extract facies information from seismic data, as the relationship between waveform associated with lithology and the specific frequency content detected in this work is not a general behavior.

\subsection{Waveform Classifier}

With this method, using the Manhattan distance, we looked for similarities between wavelets in the seismic cube, in order to classify them. We have tested different windows (e.g. $20 \mathrm{~ms}, 35 \mathrm{~ms}, 22 \mathrm{~ms}$.) around the main horizons Bur4 and Bur2 and different number of classes to be identified (e.g. 8 and 10). The maps obtained (see Fig. 15) do not show any behavior that can be correlated with the lithology of the area. No stratigraphic character or geometric feature that can be correlated with a particular sedimentary pattern (as channels bars, for example) can be inferred from them. Also no correlation between different maps could be observed. These results suggest that there is no relationship between the waveform of the seismic traces and the lithology or sedimentary environment, supporting the interpretation of those results obtained with the WBFA of the seismic data. Again, the lack of frequencies and the phase problem could be the reasons for the results obtained, as was previously described.

\section{Conclusions}

In this work, we have applied the WBFA method for facies classification at the Oritupano-A field. A power-law relationship between variance and scale was obtained for the analyzed well logs, indicating that the studied signals are nonstationary and self-affine fractals. After applying the method to a statistically significant number of wells and logs, the results also indicate that the fractal parameters: slope, intercept and fractal dimension derived from them, respond to a gradual variation in the lithological content, associated with transitional environments. In fact, the D map obtained shows sedimentation and distribution patterns associated with a deltaic plain, previously interpreted in conventional geological studies. Our results suggest that lower fractal dimensions correspond to high energy sedimentary environments, as channel zones, where thick sands were deposited. Greater D values described low energy environments, where mainly shale sediments can be found. Medium values (from 0.9 to 1.2 for the fractal dimension we calculated in this work) could be associated to the heterolithic section that corresponds to the interdistributary zone of the deltaic plane, characterized by the alternation of thin sand and shale layers. These results could be explained if the fractal dimension values are correlated to the complexity of the analyzed logs. Lower D values correspond to less complex logs that show more correlation between adjacent values and smoother profiles. These features, in turn, reflect in this kind of logs the presence of thicker sands, that characterize sandy environments. For shaly environments, a decrease in the sand thickness is expected; in the studied logs, less correlation between adjacent values is observed and, consequently, greater D values can be obtained. The intercept or $b$ WBFA parameter also discriminates the well log type. These results suggest the use of WBFA parameters as waveform derived attributes for stratigraphic characterization.

For seismic data, no relation was observed between the fractal parameters derived from the WBFA and the lithofacies. The lost of waveform information associated to lithology, due to phase and frequency problems in this set of data, could be the reason for the results obtained. This interpretation was supported by the waveform classification analysis performed on these same data, as no correspondence between waveform and paleosedimentary results was observed. 


\section{Symbol index}

$\begin{array}{ll}f: & \text { time series } \\ \varphi, \psi: & \text { wavelets } \\ C(a, e): & \text { wavelet coefficients, depending on the scale, } a, \\ & \text { and the time of appearance, } e . \\ \mathrm{C}_{\varphi}: & \text { admissibility constant } \\ \sigma: & \text { wavelet coefficients variance } \\ m: & \text { slope of the linear region of the log(variance) } \\ & \text { versus scale plot } \\ & \text { intercept of the linear region of the } \\ & \text { log(variance) versus scale plot } \\ & \text { fractal dimension obtained via equation }(5) \\ D: & \text { linear regression coefficient of the linear } \\ \mathrm{R}^{2}: & \text { adjustment }\end{array}$

Acknowledgements. We would like to thank J. Aristimuño for all the information regarding the spectral behavior of the seismic data. Thoughtful reviews by J. A. Piñuela and one anonymous referee, and the editor (A. Tarquis) comments are highly appreciated. We thank also the complete review by V. Costanzo-Álvarez and L. Trigo.

Edited by: A. Tarquis

Reviewed by: J. A. Piñuela and another anonymous referee

\section{References}

Akay, M.: Wavelets in biomedical engineering, Wavelet transforms in biomedical engineering, 23(5), 531-542, 1995.

Álvarez, G., Sansó, B., Michelena, R. J., and Jiménez, J. R.: Lithologic characterization of a reservoir using continuous-wavelet transforms, IEEE Trans. Geos. Rem. Sen., 41(1), 59-65, 2003.

Argoul, F., Arneodo, J., Elezgaray, J., Grasseau, G., and Murenzi, R.: Wavelet transform of fractal aggregates, Phys. Lett. A, 135, 6-7, 327-336, 1989.

Aristimuño, J. and Aldana, M.: Aplicación de la teoría de lógica difusa para la predicción de parámetros petrofísicos, Proceedings of the XIII Congreso Venezolano de Geofísica, Caracas, Venezuela, 1-8, 2006.

Berry, M. V.: Diffractals, J. Phys. A.: Math. Gen., 2(6), 781-797, 1979.

Brown, R. A.: Interpretation of three-dimensional seismic data, 6th edition, American Association of Petroleum Geologists (AAPG) Eds., 540pp., 2004.

Foufoula-Georgiou, E. and Kumar, P., (Eds.): Wavelets in Geophysics: Wavelets analysis and its applications, 4, Academic Press, 372pp., 1994.

Grosso, S.: Estudio Paleosedimentológico de la Unidad Hidráulica LU, Informe Petrobras Energía Venezuela, S.A, 2002.

Haber, A. and Wilk, A.: Application of new techniques in the seismic data interpretation to enhance their examination effectiveness, Acta Montanistica Slovaca, 11(1), 51-55, 2006.

Hall, M. and Trouillot, E.: Accurate stratigraphic prediction from seismic, CSEG Recorder, March Volume, 30-37, 2004.

Hewett, T. A.: Fractal distribution of reservoir heterogeneity and their influence on fluid transport, SPE Prof. Pap. 15386, Soc. Of Pet. Eng., Richardson, Tex., 15pp., 1986.
Jiménez, J. R., Peinado, A., and Michelena, R. J.: Facies recognition using wavelet-based fractal analysis on compressed seismic data, in Proc. 69th Annu. Int. Meeting Soc. Expl. Geophysics, 1922-1925, 1999.

John, A., Lake, L. W., Torres-Verdin, C., and Srinivasan, S.: Seismic facies identification and classification using simple statistic, Proc. SPE Ann. Tech. Conf. Exh., SPE 96577, 2005.

Kumar, P. and Foufoula-Georgiou, E.: Wavelet analysis in geophysics: An introduction, in: Wavelets in geophysics, edited by: Foufoula-Georgiou, E. and Kumar, P., 4, Academic Press, 1-43, 1994.

Mandelbrot, B. B.: Fractals, Freeman Eds., San Francisco, 1977.

Mengesha, Y. G.: Atmospheric boundary-layer flow over topography: data analysis and representations of topography, M.Sc. Thesis, York University, Toronto, Canada, 1999. http://www. collectionscanada.ca/obj/s4/f2/dsk2/ftp01/MQ39212.pdf, 1999.

Mosher, C., Panda, M., and Foster, D.: Wavelet-transform-based scale analysis of seismic and reservoir data, Proc. SPIE, Mathematical Methods in Geophysical Imaging V, 3453, 147-154, 1998.

Parnaud, F., Pascual, J. C., Truskowsky, I., Gallango, O., Pasalacqua, H., and Roure, F.: Petroleum geology of the central part of the Eastern Venezuelan Basin, in: Petroleum Basins of South America, edited by: Tankard, A. J., Suárez Soruco, R., and Welsink, H. J., AAPG Memoir 62, 741-756, 1995.

Percival, D. B. and Guttorp, P.: Long-memory processes, the Allan Variance and wavelets, in: Wavelets in geophysics, edited by: Foufoula-Georgiou, E. and Kumar, P., 4, Academic Press, 325344, 1994.

Pelletier, J. D. and Turcotte, D. L.: Scale-invariant topography and porosity variations in fluvial sedimentary basins, J. Geophys. Res., 101(B12), 28 165-28 175, 1996.

Porras, J. S., Vallejo, E. L., Marchal, D., and Selva, C.: Extensional folding in the Eastern Venezuela Basin: Examples from fields of Oritupano-Leona block, AAPG Annual Meeting, Houston, Texas, 10-13 March, 2002.

Rioul, O. and Vetterli, M.: Wavelets and signal processing, IEEE Sig. Proc. Magazine, 8(4), 14-38, 1991.

Ross, D. and Peterman, P.: Increased confidence in a 3-D seismic interpretation using pre-stack and post-stack seismic attributes, Proceedings of the CSEG Conference, Canada, paper 1059, 2000.

Russell, D. A., Hanson J. D., and Ott, E.: Dimension of Strange attractors, Phys. Rev. Lett., 45(14), 1175-1178, 1980.

Tebbens, S. F., Burroughs, S. M., and Nelson, E. E.: Wavelet analysis of shoreline change on the Outer Banks of North Carolina: An example of complexity in the marine sciences, Proceedings of the National Academy of Sciences of the United States of America PNAS, 99, 1, 2554-2560, 2002.

Tielen, G. J., Lulek, T., Traa, M. R. M. J., Kuzma, M., and Caspers, W. J.: The role of the Manhattan distance in antiferromagnetic ordering, Physica A, 246(1), 199-220, 1997.

Todoeschuck, J. P., Jensen, O. G., and Labonte, S.: Gaussian scaling noise model of seismic reflection sequences: Evidence from well logs, Geophysics, 55, 480-484, 1990.

Turcotte, D. L.: Fractals and Chaos in Geology and Geophysics, 2nd edition, Cambridge Uni. Press, New York, 398pp., 2007. 